\title{
Induction and anisotropy of fluorescence of reaction center from photosynthetic bacterium Rhodobacter sphaeroides
}

\author{
Gábor Sipka • Péter Maróti \\ The publisher's version is available: Photosynth Res 2016 Jan;127(1):61-8; DOI 10.1007/s11120-015-0096-y
}

\begin{abstract}
Submillisecond dark-light changes of the yield (induction) and anisotropy of fluorescence under laser diode excitation were measured in the photosynthetic reaction center of the purple bacterium Rhodobacter sphaeroides. Narrow band (1-2 nm) laser diodes emitting at 808 and $865 \mathrm{~nm}$ were used to selectively excite the accessory bacteriochlorophyll $(\mathrm{B}, 800 \mathrm{~nm})$ or the upper excitonic state of the bacteriochlorophyll dimer $\left(\mathrm{P}_{-}, 810 \mathrm{~nm}\right)$ and the lower excitonic state of the dimer $(\mathrm{P}+, 865 \mathrm{~nm})$, respectively. The fluorescence spectrum of the wild type showed two bands centered at $850 \mathrm{~nm}(\mathrm{~B})$ and $910 \mathrm{~nm}(\mathrm{P}-$ ). While the monotonous decay of the fluorescence yield at 910 $\mathrm{nm}$ tracked the light-induced oxidation of the dimer, the kinetics of the fluorescence yield at $850 \mathrm{~nm}$ showed an initial rise before a decrease. The anisotropy of the fluorescence excited at $865 \mathrm{~nm}$ (P-) was very close to the limiting value (0.4) across the whole spectral range. The excitation of both B and P- at $808 \mathrm{~nm}$ resulted in wavelength-dependent depolarization of the fluorescence from 0.35 to 0.24 in the wild type and from 0.30 to 0.24 in the reaction center of triple mutant (L131LH- M160LH-M197FH). The additivity law of the anisotropies of the fluorescence species accounts for the wavelength dependence of the anisotropy. The measured fluorescence yields and anisotropies are interpreted in terms of very fast energy transfer from ${ }_{1} \mathrm{~B}^{*}$ to ${ }_{1} \mathrm{P}$ - (either directly or indirectly by internal conversion from ${ }_{1} \mathrm{P}+$ ) and to the oxidized dimer.
\end{abstract}

\section{Introduction}

The reaction center (RC) protein of the photosynthetic bacterium Rhodobacter (Rba.) sphaeroides is designed to convert light energy into chemical potential by very fast and effective transfer of electronic excitation energy and initial electron transfer among cofactors arranged in two branches, labeled M (inactive) and L (active) forming a pseudo twofold axis of symmetry (Deisenhofer and Michel 1991; Wraight 2004). The six chlorophyll-like pigments, the special pair dimer (P) with two strongly interacting bacteriochlorophylls, the two accessory bacteriochlorophylls (B), and the two bacteriopheophytins $(\mathrm{H})$ assure (a) very fast excitation of $\mathrm{P}$ either directly by absorption of light or through energy transfer and (b) transfer of an electron from $\mathrm{P}^{*}$ to $\mathrm{H}$ within 3 ps (Zinth and Wachtveitl 2005; Jones 2009; Zhu et al. 2013). Figure 1 shows the atomic arrangement of the dimer and the two accessory bacteriochlorophylls together with their transition dipoles between the excited states and ground state. They differ in direction significantly setting the stage for orientation-dependent intraprotein energy transfer and anisotropy of fluorescence of these chromophores. 


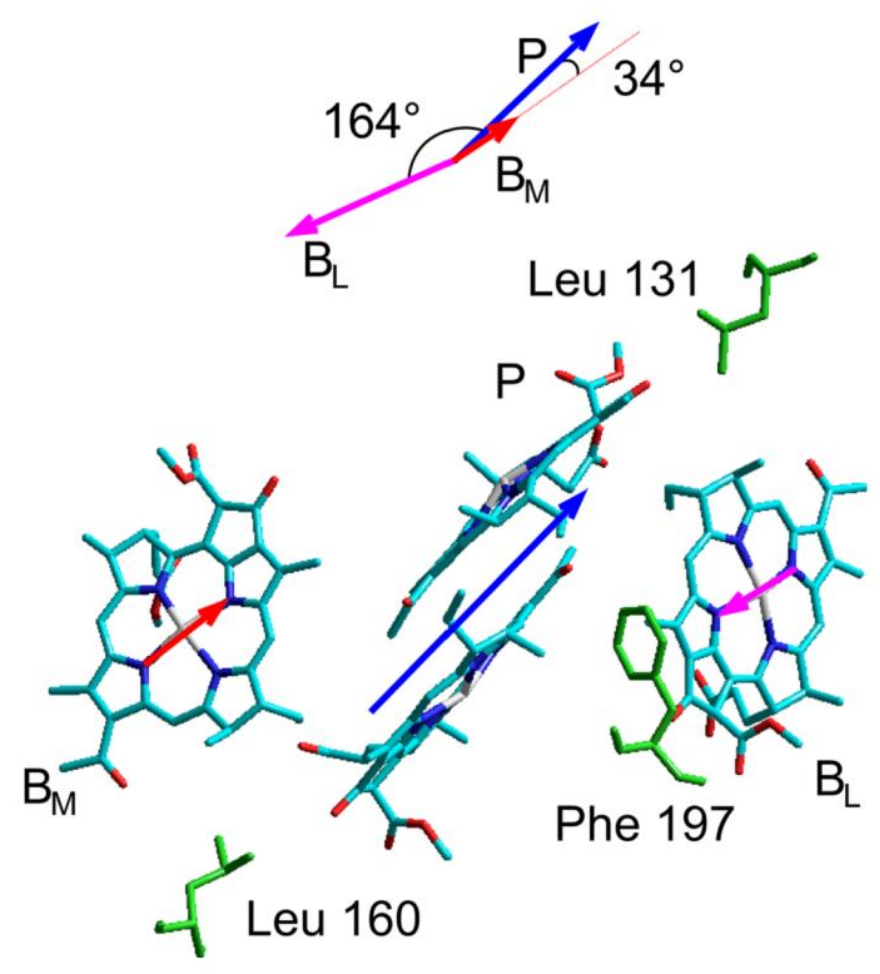

Fig. 1 The dimeric $(\mathrm{P})$ and monomeric $\left(\mathrm{BM}_{\mathrm{M}}\right.$ and $\mathrm{BL}$ ) bacteriochlorophylls without their phytol chains together with three amino acid residues targeted for mutation in the atomic structure of the wild-type reaction center of Rba. sphaeroides (PDB structure 3I4D, resolution $2.01 \mathrm{~A}^{\circ}$ ). Arrows show the projection of the $\mathrm{Q}_{\mathrm{y}}$ transition dipoles onto the plane of the figure for the accessory bacteriochlorophylls and for the lower excitonic state of the dimer (after Jonas et al. 1996). The dipoles of $\mathrm{BL}_{\mathrm{L}}$ and Bм are at angles 164 and 34 relative to that of the dimer, respectively (top)

The cofactors can be characterized and identified by specific bands of the near-IR absorption and fluorescence spectra. The absorption band of $\mathrm{H}$ is at $760 \mathrm{~nm}$, and the absorption and fluorescence bands due to the $\mathrm{Qy}$ transition of both bacteriochlorophyll monomers (B) are at 800 and $850 \mathrm{~nm}$, respectively. The two bacteriochlorophylls of the dimer have extensively delocalized excitations that are close to symmetric $(\mathrm{P}+)$ and antisymmetric $(\mathrm{P}-)$ combinations of monomeric excitations. The absorption band attributed to transition to $\mathrm{P}+$ is located at $810 \mathrm{~nm}$ and overlaps the band of B at $800 \mathrm{~nm}$. At low temperature, it appears as a shoulder near $810 \mathrm{~nm}$ (Warshel and Parson 1991; Parson and Warshel 2009). The transition to P-state is characterized by a strong band at $865 \mathrm{~nm}$. The transition moment of the $\mathrm{P}+$ band is nearly orthogonal to that of $\mathrm{P}-$-, and the dipole strength is about 10 times larger than that of $\mathrm{P}$.. The observed fluorescence comes from $\mathrm{P}$ - and gives a band at $910 \mathrm{~nm}$. A number of spectroscopic studies using ultrafast pumpprobe absorption and fluorescence method (Jonas et al. 1996; Arnett et al. 1999), fluorescence up-conversion (Stanley et al. 1996), delayed fluorescence (Onidas et al. 2013), single RC crystals (Huang et al. 2012), linear dichroism (Breton 1985), and hole burning (Reddy et al. 1993) supported by theoretical calculations (Warshel and Parson 1991; Scherer et al. 1994) have addressed the interpretation of energetics and dynamics of excitation energy- and electron transfer. They revealed some degree of coupling between the cofactors among which the coupling with the excitonic states of the dimer has the largest impact. It is well documented that $\mathrm{P}$ - state is mixed with charge transfer states (Boxer et al. 1989), and P+is mixed with accessory bacteriochlorophyll states leading to several transitions forming the absorption band at $800 \mathrm{~nm}$. Due to the strong coupling and ultrafast energy transfer ( $100 \mathrm{fs}$ ) between $\mathrm{B}$ and $\mathrm{P}$, it is proposed that there is a transfer from a $\mathrm{B} / \mathrm{P}+$ mixed state to a state of mostly Pcharacter (Arnett et al. 1999) rather than via separate steps (B* $\rightarrow$ P+ energy transfer followed by P+ $\rightarrow$ P- internal conversion) (Jonas et al. 1996). To obtain more information on the intraprotein excitation energy transfer between $\mathrm{B}$ and $\mathrm{P}$, we performed (quasi) steady-state fluorescence induction and anisotropy spectroscopy on isolated RCs from wild-type and triple mutant L131LH-M160LH-M197FH strains of Rba. sphaeroides. The mutation affects the energetics of the dimer (the redox midpoint potential is elevated (Lin et al. 1994), and the primary charge pair P+QA - is destabilized by about $200 \mathrm{meV}$ (Onidas et al. 2013)) and the coupling between B and $\mathrm{P}$ because the mutation sites are in close vicinity of the cofactors (Fig. 1). The experiments reported here were carried out under physiological conditions including single and low pulse energy that avoids saturation and 
multiple excitation of the RC. By analyzing the spectra of induction and anisotropy of fluorescence of different RCs after selective excitation, we deduce below that (quasi) steadystate measurements with adequate structural information can offer insight into the world of ultrafast processes.

\section{Materials and methods}

The cultivation of the carotenoid-containing strain 2.4.1 of Rba. sphaeroides and the isolation and the purification of the RC protein were described earlier (Maróti and Wraight 1988). The construction of the triple mutant strain L131LH-M160LH-M197FH, the mutagenesis procedures, growth conditions for mutant cells, as well as the RC preparation have been described previously (Spitz et al. 2005). The photochemical function of the RC was characterized by the near-infrared steady-state absorbance spectrum and by the flash-induced $\mathrm{P} / \mathrm{P}+$ absorption change at $860 \mathrm{~nm}$ performed on a kinetic spectrophotometer of local design. The purity of the RC preparation is characterized by the $\mathrm{OD}(280) / \mathrm{OD}(802)$ ratio that was less than 1.3 in our experiments. The secondary quinone activity of the RC remaining after preparation was inhibited by the addition of $100 \mu \mathrm{M}$ terbutryn.

\section{Fluorescence induction}

The kinetics and yield of the RC fluorescence on illumination with rectangular profile were recorded by a homebuilt spectrofluorometer with laser diode excitation of high (1-2 W) power (Roithner LaserTechnik) (Maróti 2008). The emission wavelengths and bandwidths of the laser diodes were $808( \pm 0.5 \mathrm{~nm})$ and $865( \pm 1 \mathrm{~nm})$. The laser beam was expanded and homogenized by ground glass at the cuvette to avoid mixing of kinetics due to heterogeneous illumination. The laser intensity was attenuated by calibrated neutral density filters. The rise time of the laser excitation was much less than the time resolution of the device $(\sim 1 \mu \mathrm{s})$, and the flash of the laser diode formed the shape of a step function with good approximation. The fluorescence of the RC was detected at 90 o with respect to the excitation light through an IR monochromator (Jobin- Yvon H-20 with a concave holographic grating) by a redsensitive photomultiplier tube (Hamamatsu R3310-03) that was cooled down to -30 C (Photocool PC 410CE; Products for Research) to reduce the dark current by 2-3 orders of magnitude. The kinetic trace of the fluorescence was divided by that of the laser diode emission to obtain the relative yield of the fluorescence.

\section{Fluorescence anisotropy}

The wavelength-resolved anisotropy of laser-diode-excited fluorescence was measured with standard right-angle geometry (Fig. 2). The polarization of the partially polarized exciting laser diode was achieved by a Glan-Taylor polarizer $(\mathrm{P})$. The fluorescence from the sample was polarized by a Glan-Thompson polarizer (A) either perpendicular or parallel to the direction of the polarizer. The analyzer could be rotated by the New Focus rotary stage (8401) and controlled by PC equipped with a New Focus Intelligent Picomotor Driver. At the corner of the right angle, the cuvette in a massive brass block contained the RC solution thermostated at room temperature. The fluorescence was excited by linearly polarized laser light with either vertical $(\mathrm{V})$ or horizontal $(\mathrm{H})$ direction of the electric vector referred to the laboratory system. The anisotropy of the fluorescence, $r$, was determined by

$$
r=\frac{I_{\mathrm{VV}}-G \cdot I_{\mathrm{VH}}}{I_{\mathrm{VV}}+2 G \cdot I_{\mathrm{VH}}},
$$

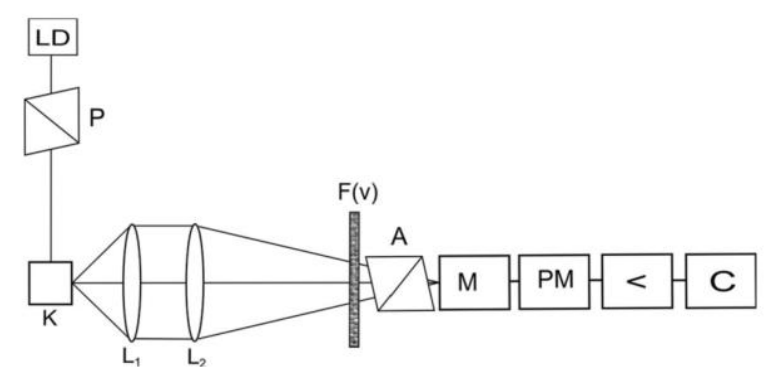

Fig. 2 Schematic arrangement of the apparatus used to excite and detect the polarized fluorescence. Notations: LD: laser diode of wavelengths 806 or $865 \mathrm{~nm}$, P: Glan-Taylor polarizer, K: $191 \mathrm{~cm}$ thermostated quartz cuvette with the sample, $\mathrm{L}_{1}$ and L2: lens system for collimation of the fluorescence, F: combination of infrared transmitting filters, A: Glan-Thompson polarizer, M: infrared monochromator, PM: cooled photomultiplier tube, <: electric amplifier, and C: computer 
where $\mathrm{G}=\mathrm{Inv} / \mathrm{IHн}$ is the instrument-factor. The two letters in the subscript of the fluorescence intensity, I, denote the directions of the polarization of the excitation (first letter) and emission (second letter). The instrument-factor, $\mathrm{G}$, describes the contribution of all factors (e.g., monochromator with holographic grid) that cause depolarization and should be separated from that due to the sample. The G-factor was determined prior to and occasionally during the measurement. The fluorometer was calibrated by scattering the light of a tungsten lamp in ludox solution and by detection of the fluorescence of BChl or IR806 dyes in castrol oil excited by laser diode (Ebrey and Clayton 1969).

\section{Results}

The fluorescence of the bacterial RC can be selectively excited by monochromatic light of laser diodes adequate to the absorption bands of the chromophores. Using diode lasing at $808 \mathrm{~nm}$, both B and the upper exciton state of the dimer in wild-type RC can be directly excited resulting in two well-defined fluorescence bands at 850 and 910 $\mathrm{nm}$ corresponding to radiative transitions from $1 \mathrm{~B}^{*}$ and from $1 \mathrm{P}_{-}$(the lower exciton state of the dimer) to the ground state, respectively (Fig. 3a). If the RC is chemically oxidized by excess ferricyanide before the excitation, the band characteristic of the dimer disappears (Fig. 3b). The oxidation of the dimer can be achieved progressively by light excitation. The $\mathrm{P} \rightarrow \mathrm{P}+$ transition during laser diode excitation is reflected by drop of the yield of the dimer fluorescence band at $910 \mathrm{~nm}$ as a result of loss of excitable P (Fig. 4a). The phenomenon is similar to characteristic changes of bacteriochlorophyll fluorescence yield in intact cells upon dark $\rightarrow$ light transition (fluorescence induction) (Clayton 1966; Osva 'th et al. 1996) that has gained a wide field of interest and applications (Koblizek et al. 2005; Kocsis et al. 2010; Asztalos et al. 2012; Kis et al. 2014). The rate constant of decay of the fluorescence yield is controlled by the intensity of the laser diode. The small fluorescence signal remained after the photooxidation of the dimer can be attributed to impurities of the isolated $\mathrm{RC}$.
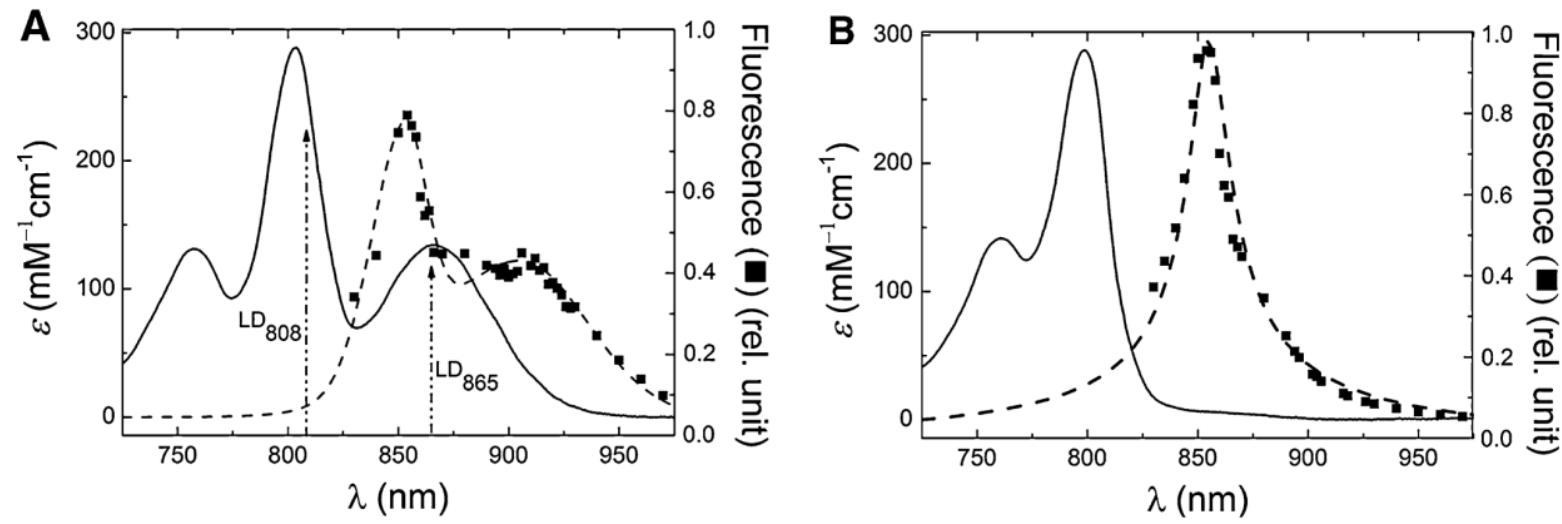

Fig. 3 Near-IR absorption and fluorescence spectra of native (panel a) and chemically oxidized (panel b) photosynthetic reaction center from Rba. sphaeroides 2.4.1 at room temperature. The absorption and fluorescence bands of the dimer disappear upon oxidation of the RC. Conditions: 2-10 $\mu \mathrm{M}$ RCs, $0.03 \%$ LDAO detergent, $10 \mathrm{mM}$ Tris buffer, and $\mathrm{pH} 8.0$ (panel a) and $\sim 40 \mathrm{mM}$ potassium ferricyanide (panel b)

in intact cells upon dark $\rightarrow$ light transition (fluorescence induction) (Clayton 1966; Osva 'th et al. 1996) that has gained a wide field of interest and applications (Koblizek et al. 2005; Kocsis et al. 2010; Asztalos et al. 2012; Kis et al. 2014). The rate constant of decay of the fluorescence yield is controlled by the intensity of the laser diode. The small fluorescence signal remained after the photooxidation of the dimer can be attributed to impurities of the isolated RC. The kinetics of the induction shows remarkable changes when the emission is detected not at $910 \mathrm{~nm}$ but at $850 \mathrm{~nm}$ (Fig. 4b). At this wavelength, the contribution of B dominates and determines the observed fluorescence of the RC (Fig. 4c). After the initial rise, the fluorescence levels off at relatively high intensity. The opposite shapes of fluorescence kinetics with identical fall and rise times at 910 and $850 \mathrm{~nm}$, respectively, serve as clear cut evidence for the electronic excitation energy transfer from $1 \mathrm{~B}^{*}$ to the dimer. The more general and demonstrative connection between the spectral and kinetic changes of the fluorescence induction of wildtype RC is shown in a 3D relief map (Fig. 4d). The triple mutation of the amino acids between $\mathrm{P}$ and $\mathrm{B}$ does not change the near-IR absorption spectrum of the RC, but modifies the spectrum and the induction kinetics of the fluorescence significantly. While the yield of the B fluorescence at $850 \mathrm{~nm}$ decreases dramatically, the yield of the dimer fluorescence at $910 \mathrm{~nm}$ increases by a factor of two (Fig. 4c). The rate constant of light utilization for charge separation measured as the decay constant of the fluorescence induction at $910 \mathrm{~nm}$ is not modified upon mutation 
(Fig. 4a). However, the kinetics detected at $850 \mathrm{~nm}$ become significantly different (see the convex/concave difference in Fig. 4b). The anisotropy of the dimer fluorescence upon direct excitation of the $865 \mathrm{~nm}$ band is high (very close to the theoretical maximum of +0.4 ) both for wild-type and mutant RC and shows no wavelength dependence (Fig. 5b, d). Similarly, high and constant fluorescence anisotropies can be observed if the dimer is chemically oxidized by ferricyanide (Fig. 5a). The anisotropy spectrum will change if the lower excitonic level of the dimer is not directly excited. The anisotropy of fluorescence from B will be mixed with those of the dimer excited both directly and indirectly. The excitation energy transfer from ${ }_{1} \mathrm{~B} *$ to ${ }_{1} \mathrm{P}$ - depolarizes the indirectly excited dimer fluorescence. According to the law of additivity of anisotropies, the observed anisotropy will be the sum of the anisotropies weighted by the fluorescence intensities of the species. The calculated spectrum of fluorescence anisotropy performs a smoothed step function with descending end at longer wavelengths both for wildtype and mutant RC (Fig. 5a, c). The selective excitation of the Qy transitions of B and P keeps the anisotropy at elevated level below $850 \mathrm{~nm}$ which is not the case if other cofactors and other transitions are simultaneously excited as reported in (Ebrey and Clayton 1969).

\section{Discussion}

The observed spectral and temporal changes of the yield and anisotropy of the RC fluorescence during induction will be discussed based on energy level diagram of the two chromophores ( $\mathrm{P}$ and $\mathrm{B}$ ) that are relevant to both the energy and electron transfer processes under our conditions (Fig. 6).

\section{Yield of fluorescence}

Upon excitation of the $800 \mathrm{~nm}$ absorption band, two distinct fluorescence bands at 850 and $910 \mathrm{~nm}$ appeared that could be attributed to radiative transfer from excited ${ }_{1} \mathrm{P}^{*}$ and ${ }_{1} \mathrm{~B} *$ states to the ground state, respectively. The quantum yield of the dimer fluorescence is very low: 410-4 (Zankel et al. 1968) due to the high rate constant of the photochemistry (charge separation). Similarly, low value can be expected for the B fluorescence because (1) the dimer $(1 \mathrm{P}+)$ absorption near $810 \mathrm{~nm}$ accounts for roughly one-quarter of the B absorption around $800 \mathrm{~nm}$ and (2) the fluorescence intensities of the two fluorescence bands are in the same orders of magnitude in wild-type RC (Fig. 3). Although the exact rate constant of the energy transfer from ${ }_{1} \mathrm{~B} *$ to $1 \mathrm{P}$ via ${ }_{1} \mathrm{P} \mathrm{p}$ is debated (Jonas et al. 1996; Stanley et al. 1996; Arnett et al. 1999), it should be at least 10 times larger than that of the charge separation. To get about similar quantum yields of fluorescence for $\mathrm{B}$ and $\mathrm{P}$, one has to assume at least 10 times larger radiative rate constant for $\mathrm{B}$ than for $\mathrm{P}$ in the wild-type $\mathrm{RC}$. The intraprotein chemical surrounding and energetic coupling may account for this difference. 

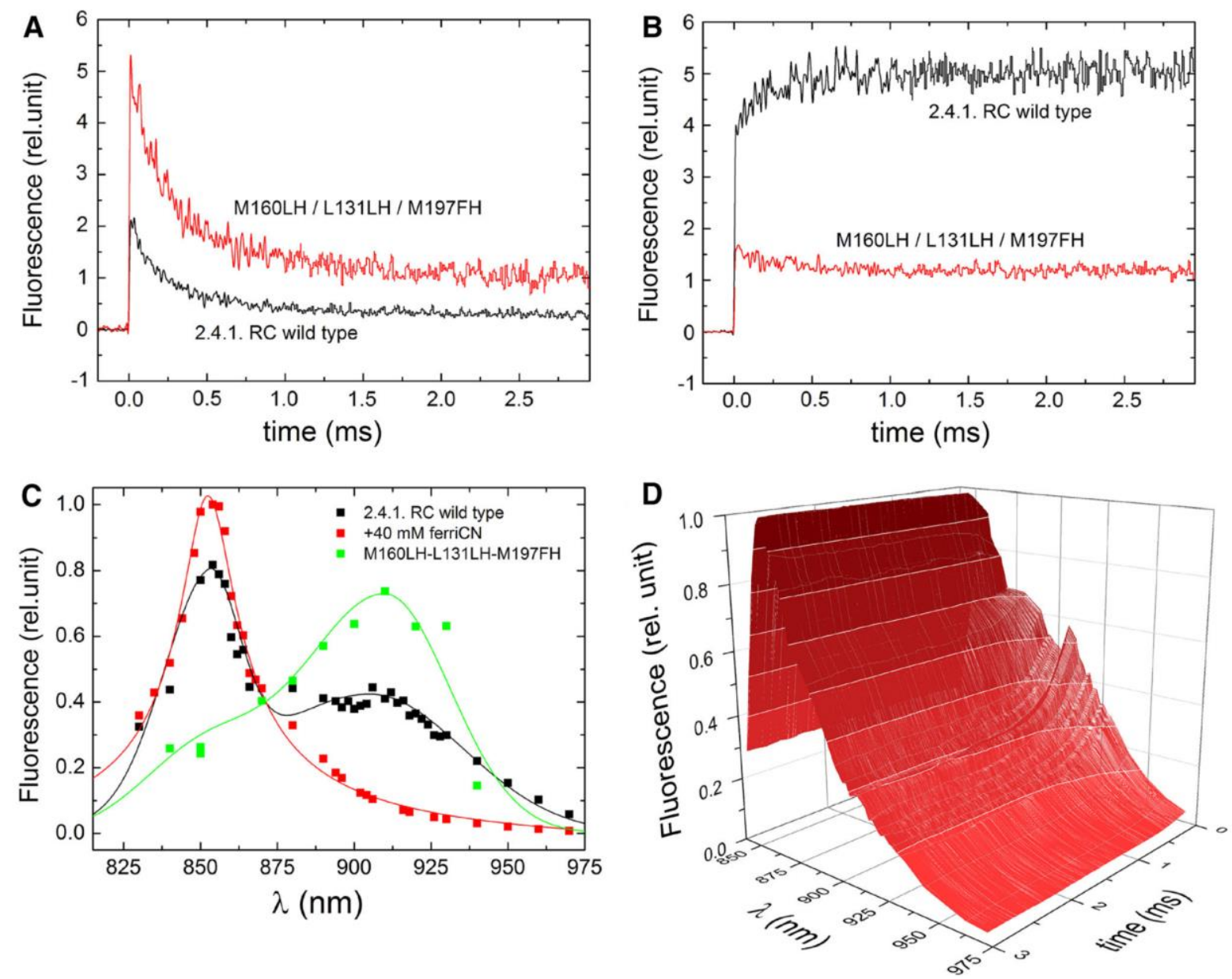

Fig. 4 Spectral and kinetics changes of fluorescence induction evoked by $808 \mathrm{~nm}$ laser diode excitation in wild-type and triplemutated (L131LH-M160LH-M197FH) reaction centers of Rba. sphaeroides. Kinetics of fluorescence induction observed at $910 \mathrm{~nm}$ (panel a) and at $850 \mathrm{~nm}$ (panel b) for WT (black) and triple mutant (red) RCs. Fluorescence spectra of WT (black dots) and mutant (green dots) RCs in reduced and (chemically) oxidized states of the dimer (red dots) (panel c).

Quasi 3D demonstration of the spectrum and kinetics of fluorescence in wild-type RC (panel d)

The finding was interesting that the yield of the B fluorescence did not change dramatically upon oxidation of the dimer: a slight increase was detected only. If $\mathrm{P}$ is oxidized (either chemically or progressively by light) then no $1 \mathrm{~B}^{*}$ to ${ }_{1} \mathrm{P}^{*}+$ energy transfer will take place, i.e., much larger yield of $\mathrm{B}$ fluorescence will be expected. However, this does not occur, probably because of the appearance of another deactivating (loss) process with similar rate constant as the $\mathrm{B}^{*} \rightarrow{ }_{1} \mathrm{P}^{*}+$ energy transfer. As $1 \mathrm{~B}^{*}$ and ${ }_{1} \mathrm{P}+$ ox (the upper excitonic level of the oxidized dimer) are approximately isoenergetic states and are closely located, strong energetic coupling and therefore high transfer rate can be expected that keeps the yield of B fluorescence on the observed low level (Fig. 6). 

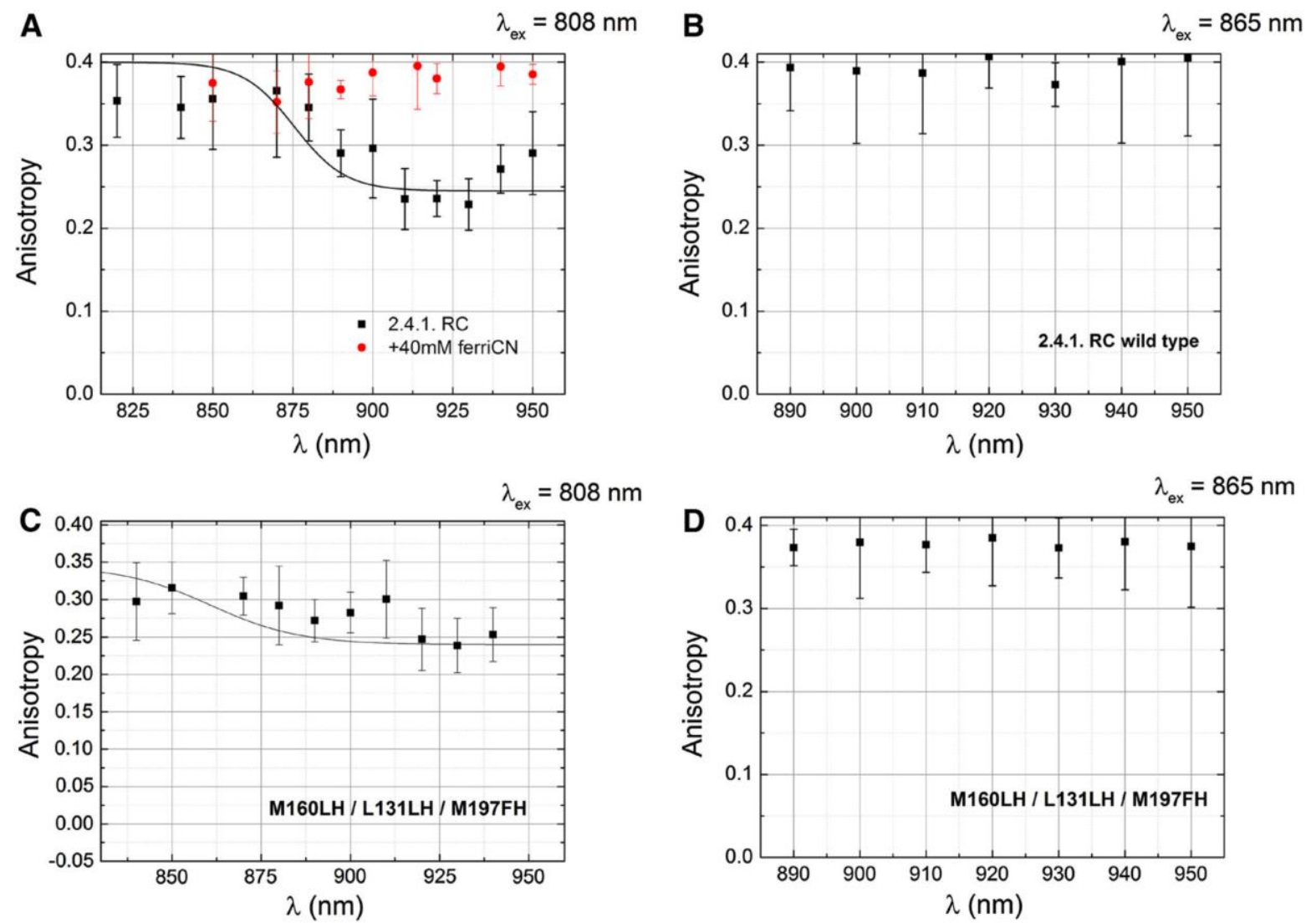

Fig. 5 Anisotropy spectra of fluorescence excited at 808 and $865 \mathrm{~nm}$ by laser diodes in wild-type (panels a and b) and triple mutant (panels c and d) RCs of Rba. sphaeroides. Upon excitation at $808 \mathrm{~nm}$, the fluorescence originates from $\mathrm{B}^{*}$ and $\mathrm{P}-$, and therefore, the observed anisotropy is the sum of anisotropies of the species weighted by their fluorescence intensities determined from Gaussian decomposition of the fluorescence spectra (Fig. 3.). The calculated anisotropies (solid lines in panels a and c) are close to the data points within the error of the measurement

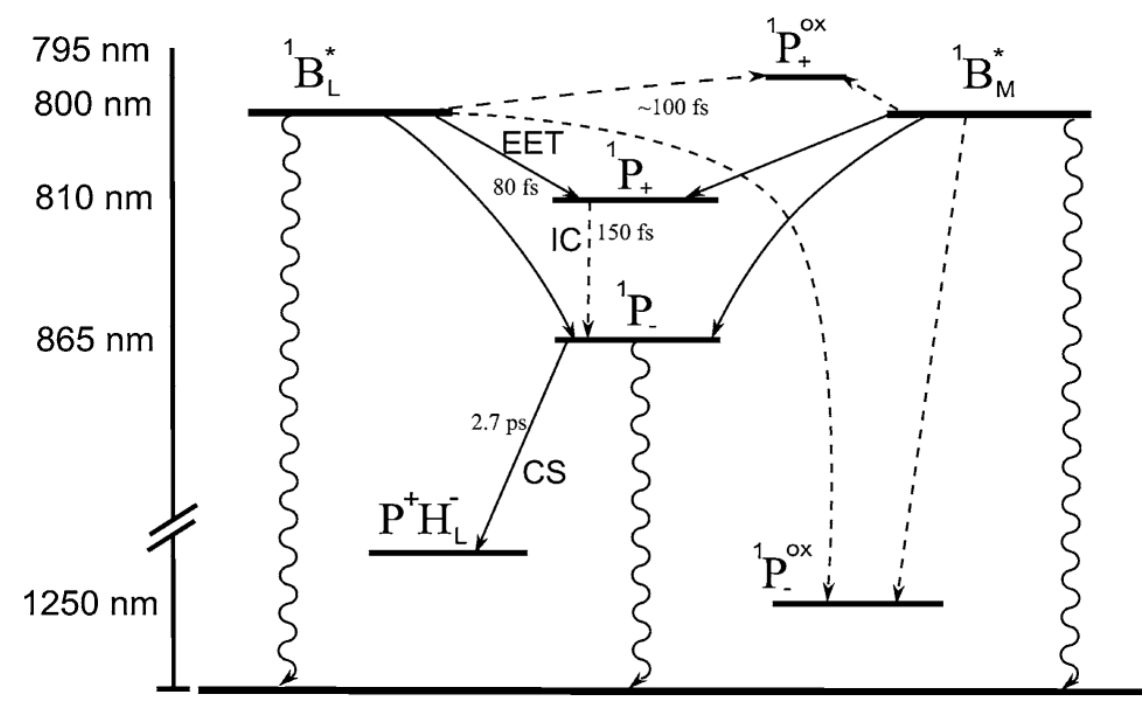

Fig. 6 Schematic energy level diagram of singlet excited bacteriochlorophyll monomers ( $1 \mathrm{~B}_{\mathrm{L}} *$ and $\left.{ }_{1} \mathrm{BM}_{\mathrm{M}} *\right)$, lower $(1 \mathrm{P}-)$ and upper $\left({ }_{1} \mathrm{P}+\right)$ excitonic levels of the dimer in the reduced $\left({ }_{1} \mathrm{P}\right)$ and oxidized $\left({ }_{1} \mathrm{Pox}\right)$ states and charge separated $\mathrm{P}+\mathrm{HL}-\mathrm{state}$. The scale of energy levels of the states is not proportional. The possible pathways of excitation energy transfer (EET), internal conversion (IC), charge separation (CS), and fluorescence emission (wavy arrow) with approximate time half times are also 


\section{Anisotropy of fluorescence}

Upon excitation of the lower excitonic level of the dimer ( $\left.\mathrm{P}_{-}\right)$by light of $865 \mathrm{~nm}$ wavelength, the measured fluorescence anisotropy was very close to the maximum theoretical value of +0.4 all over the spectral range covering the fluorescence spectrum. This observation signifies a single transition (or two distinct but parallel transitions) and not a pair of degenerate transitions. The single CD band coincident with the $870 \mathrm{~nm}$ absorption band supports this conclusion (Sauer and Austin 1978). Similarly, fluorescence anisotropy close to the limiting value was obtained by excitation at $808 \mathrm{~nm}$ if the dimer was oxidized. Consequently, the absorption and emission dipole moments of the monomeric bacteriochlorophyll (B) should be (close to) parallel. If the dimer is not oxidized, then both $\mathrm{P}$ and $\mathrm{B}$ will emit fluorescence, and the observed anisotropy will be the sum of anisotropies of the components weighted by the corresponding fluorescence intensities. The fluorescence from $\mathrm{B}$ has anisotropy $\mathrm{r}=$ $\mathrm{r} 0=+0.4$ because of direct excitation. The dimer fluorescence comes from state ${ }_{1} \mathrm{P}$ that can be populated either from $1 \mathrm{P}^{*}+\left(\right.$ anisotropy $\mathrm{r}=-0.2$, because $1 \mathrm{P}+$ makes a large angle $(\sim 90 \mathrm{o})$ with $\left.{ }_{1} \mathrm{P}-\right)$ or from ${ }_{1} \mathrm{BL}^{*}$ and ${ }_{1} \mathrm{BM}^{*}$ by energy transfer (anisotropy $r=r_{0}\left\{\left(\frac{3 \cdot \cos ^{2} \alpha_{\mathrm{PL}}-1}{2}\right)+\left(\frac{3 \cdot \cos ^{2} \alpha_{\mathrm{PM}}-1}{2}\right)\right\}$, here aPL and aPM denote the angles between transitions of $\mathrm{P}$ and $\mathrm{BL}$ and $\mathrm{P}$ and $\mathrm{BM}$, respectively (see Fig. 1)). To explain the fairly high measured value of the anisotropy of the dimer fluorescence, a significant portion of energy transfer from $1 \mathrm{~B} *$ should directly target the $1 \mathrm{P}^{*}+$ state. Different kinetic models including several steps (combination of energy transfer and internal conversion) between several states $\left(1 \mathrm{~B}^{*},{ }_{1} \mathrm{P}^{*}+,{ }_{1} \mathrm{P}^{*}\right.$ and $\left.\mathrm{P}+\mathrm{H}_{-}\right)$may account for this possibility (Jonas et al. 1996; Arnett et al. 1999). The wavelength dependence of the anisotropy of the fluorescence based on our crude model gives acceptable agreement with the experiment.

\section{Triple mutant}

The 2-acetyl and the 9-keto groups of both $\mathrm{PM}_{\mathrm{M}}$ and $\mathrm{PL}_{\mathrm{L}}$ in the dimer are engaged in a hydrogen-bond with the surrounding protein. The L131LH-M160LH-M197FH mutation modifies the hydrogen-bonding pattern of the dimer by introduction of three His residues capable of $\mathrm{H}$-bond formation. The increase of the number of H-bonds in the mutant increases the redox midpoint potential of the dimer by about $200 \mathrm{meV}$ (Lin et al. 1994), the intensity of the delayed fluorescence, and destabilizes the P+QA - charge pair by $200 \mathrm{meV}$ at pH 8 (Onidas et al. 2013). Our experiments indicated, however, that the major (prompt) fluorescence properties of the dimer, i.e., the spectrum, the orientation and strength of transition dipoles, the magnitude of the Stokes-shift, and the yield of fluorescence were hardly modified by the mutation. On the other hand, a significant drop of the yield of fluorescence related to the monomeric bacteriochlorophyll was experienced. The decrease of the yield could include either an increase of the rate of electronic excitation energy transfer to $1 \mathrm{P}$ or a decrease of the intrinsic rate constant of radiation of $1 \mathrm{~B} *$ relative to those in wild-type $\mathrm{RC}$. The mutation of amino acids between $\mathrm{P}$ and $\mathrm{B}$ can affect the coupling between the chromophores resulting in changes of the fluorescence properties of B and excitation energy transfer to the dimer.

\section{Acknowledgments}

This paper is dedicated to the memory of Dr. Colin A. Wraight (1945-2014) whose pioneering contributions to spectroscopy of bacterial RC have inspired many investigators (including PM). Thanks to Drs. P. Sebban and D. Onidas (University of Paris XI, Faculte'd'Orsay, France) for the mutant, to Dr. G. Laczkó (University of Szeged) for stimulating discussions, and to TÁMOP 4.2.2.A-11/1KONV-2012-0060, TA ' MOP 4.2.2.B, OTKA K116834, and COST Actions CM1306 for financial support.

\section{References}

Arnett DC, Moser CC, Dutton PL, Scherer NF (1999) The first eventsin photosynthesis: electronic coupling and energy transferdynamics in the photosynthetic reaction center from Rhodobacter sphaeroides. J Phys Chem 103:2014-2032

Asztalos E, Sipka G, Kis M, Trotta M, Maróti P (2012) The reactioncenter is the sensitive target of the mercury(II) ion in intact cellsof photosynthetic bacteria. Photosynth Res 112:129-140

Boxer SG, Goldstein RA, Lockhart DJ, Middendorf TR, Takiff L(1989) Excited states, electron-transfer reactions, and intermediates in bacterial photosynthetic reaction centers. J Phys Chem93:8280-8294

Breton J (1985) Orientation of the chromophores in the reactioncenter of Rhodopseudomonas viridis. Comparison of lowtemperature linear dichroism spectra with a model derived fromX-ray crystallography. Biochim Biophys Acta 810:235-245 
Clayton RK (1966) Relations between photochemistry and fluorescence in cells and extracts of photosynthetic bacteria. PhotochemPhotobiol 5:807-821

Deisenhofer J, Michel H (1991) High-resolution structures ofphotosynthetic reaction centers. Annu Rev Biophys BiophysChem 20:247-266

Ebrey TG, Clayton RK (1969) Polarization of fluorescence frombacteriochlorophyll in castor oil, in chromatophores and as P870in photosynthetic reaction centers. Photochem Photobiol10:109-117

Huang L, Ponomarenko N, Wiederrecht GP, Tiede DM (2012)Cofactor-specific photochemical function resolved by ultrafastspectroscopy in photosynthetic reaction center crystals. ProcNatl Acad Sci 109:4851-4856

Jonas DM, Lang MJ, Nagasawa Y, Joo T, Fleming GR (1996) Pumpprobe polarization anisotropy study of femtosecond energytransfer within the photosynthetic reaction center of Rhodobactersphaeroides R26. J Phys Chem 100:12660-12673

Jones MR (2009) The petite purple photosynthetic powerpackBiochem Soc Trans 37:400-407

Kis M, Asztalos E, Sipka G, Maróti P (2014) Assembly of photosyntheticapparatus in Rhodobacter sphaeroides as revealed by functionalassessments at different growth phases and in synchronized andgreening cells. Photosynth Res 122(3):261-273

Koblizek M, Shih JD, Breitbart SI, Ratcliffe EC, Kolber ZS, HunterCN, Niederman RA (2005) Sequential assembly of photosynthetic units in Rhodobacter sphaeroides as revealed by fastrepetition rate analysis of variable bacteriochlorophyll a fluorescence. Biochim Biophys Acta 1706:220-231

Kocsis P, Asztalos E, Gingl Z, Maróti P (2010) Kinetic bacteriochlorophyll fluorometer. Photosynth Res 105:73-82

Lin X, Murchison HA, Nagarajan V, Parson WW, Allen JP, WilliamsJC (1994) Specific alteration of the oxidation potential of theelectron donor in reaction centers from Rhodobacter sphaeroides. Proc Natl Acad Sci USA 91:10265-10269

Maróti P (2008) Kinetics and yields of bacteriochlorophyll fluorescence: redox and conformation changes in reaction center ofRhodobacter sphaeroides. Eur Biophys J 37:1175-1184

Maróti P, Wraight CA (1988) Flash-induced H+ binding by bacterialphotosynthetic reaction centers: comparison of spectrophotometric and conductimetric methods. Biochim Biophys Acta934:314-328

Onidas D, Sipka G, Asztalos E, Maróti P (2013) Mutational control ofbioenergetics of bacterial reaction center probed by delayedfluorescence. Biochim Biophys Acta Bioenerg 1827:1191-1199

Osva 'th Sz, Laczkó G, Sebban P, Maróti P (1996) Electron transfer inreaction centers of Rhodobacter sphaeroides and Rhodobactercapsulatus monitored by fluorescence of the bacteriochlorophylldimer. Photosynth Res 47:41-49

Parson WW, Warshel A (2009) Mechanism of charge separation inpurple bacterial reaction center. In: Hunter CN, Daldal F,Thurnauer M, Beatty JT (eds) Advances in photosynthesis andrespiration: the purple phototrophic bacteria. Springer, Dordrecht, pp 355-377

Reddy NRS, Kolaczkowski SV, Small GJ (1993) Nonphotochemicalhole burning of the reaction center of Rhodopseudomonasviridis. J Phys Chem 97(26):6934-6940

Sauer K, Austin L (1978) Bacteriochlorophyll-protein complexesfrom light-harvesting antenna of photosynthetic bacteria. Biochemistry 17:2011-2019

Scherer POJ, Fischer SF, Lancaster CRD, Fritzsch G, Schmidt S, ArltT, Dressler K, Zinth W (1994) Chem Phys Lett 223:110-115

Spitz JA, Derrien V, Baciou L, Sebban P (2005) Specific triazineresistance in bacterial reaction centers induced by a singlemutation in the QA protein pocket. Biochemistry 44:1338-1343

Stanley RJ, King B, Boxer SG (1996) Excited state energy transferpathways in photosynthetic reaction centers. 1. Structuralsymmetry effects. J Phys Chem 100:12052-12059

Warshel A, Parson WW (1991) Computer simulations of electrontransfer reactions in solution and in photosynthetic reactioncenters. Annu Rev Phys Chem 42:279-309

Wraight CA (2004) Proton and electron transfer in the acceptorquinone complex of photosynthetic reaction centers fromRhodobacter sphaeroides. Front in Biosci 9:309-337

Zankel KL, Reed DW, Clayton RK (1968) Fluorescence andphotochemical quenching in photosynthetic reaction centers Proc Natl Acad Sci USA 61:1243-1249

Zhu J, van Stokkum IHM, Paparelli L, Jones MR, Groot ML (2013)Early bacteriopheophytin reduction in charge separation inreaction centers of Rhodobacter sphaeroides. Biophys J104:2493-2502

Zinth W, Wachtveitl J (2005) The first picoseconds in bacterialphotosynthesis-ultrafast electron transfer for the efficientconversion of light energy. Chem Phys Chem 6:871-880 